\title{
Odeseizar la educación y la cooperación para el desarrollo en España: una mirada internacional a la AOD y al ODS 4
}

\author{
María-Jesús Martínez-Usarralde \\ Carmen Lloret-Catalá \\ Universitat de València. España. \\ m.jesus.martinez@uv.es \\ m.carmen.lloret@uv.es
}

Recibido: 15/5/2019

Aceptado: 5/12/2019

Publicado: 20/7/2020

\section{Resumen}

El presente artículo pretende centrar su atención en la política sectorial de la AOD vinculada a la educación desde la ingeniería cosmopolita de los objetivos de desarrollo sostenible que han permitido odeseizar las políticas mundiales (Barandiarán, 2017), de las que la cooperación y la educación constituyen dos objetos de análisis e investigación. Para ello, sus objetivos se centrarán en revisar el caso español del binomio AOD y educación, y cómo, estrechamente vinculado a la ortodoxia política mundial homogeneizadora que proviene de manera intencionalmente manifiesta por parte de los organismos internacionales, la AOD en la actualidad y en este ámbito específico se ha canalizado y transmutado en el cuarto objetivo de desarrollo sostenible (ODS 4). La metodología utilizada se basa en técnicas cualitativas centradas en el análisis documental y de contenido sobre AOD y ODS 4 procedente de organismos internacionales e informes oficiales. En las conclusiones se pretende articular el discurso inicial de la vinculación existente entre la política actual española del ODS 4 y la de la AOD, al tiempo que se explica su proyección futura más inmediata.

Palabras clave: cooperación para el desarrollo; educación; ayuda oficial para el desarrollo (AOD); objetivos de desarrollo sostenible (ODS); educación internacional

Resum. Odeseïtzar l'educació i la cooperació per al desenvolupament a Espanya: una mirada internacional a l'AOD $i$ a l'ODS 4

Aquest article pretén centrar l'atenció en la política sectorial de l'AOD vinculada a l'educació des de l'enginyeria cosmopolita dels objectius de desenvolupament sostenible que han permès odeseïtzar les polítiques mundials (Barandiarán, 2017), de les quals la cooperació i l'educació constitueixen dos objectes d'anàlisi i d'investigació. Per fer-ho, els objectius perseguits se centraran a revisar el cas espanyol del binomi AOD i educació, i com, estretament vinculat a l'ortodòxia política mundial homogeneïtzadora que prové de manera intencionada manifesta per part dels organismes internacionals, l'AOD en l'actualitat i en aquest àmbit específic s'ha canalitzat i ha transmutat en el quart objectiu de desenvolupament sostenible (ODS 4), centrat també en l'educació. La metodologia utilitzada es basa en tècniques qualitatives centrades en l'anàlisi documental i de contingut sobre AOD 
i ODS 4 procedent d'organismes internacionals i d'informes oficials. En els resultats i en les conclusions es pretén articular el discurs inicial de la vinculació existent entre la política actual espanyola de l'ODS 4 i la de l'AOD, alhora que se n'explica la projecció futura més immediata.

Paraules clau: cooperació per al desenvolupament; educació; ajuda oficial per al desenvolupament (AOD); objectius de desenvolupament sostenible (ODS); educació internacional

Abstract. The odeseization of education and development cooperation in Spain: An international look at $O D A$ and $S D G 4$

This article examines the sectorial policy of official development assistance (ODA) linked to education through the cosmopolitan engineering of the Sustainable Development Goals (SDG) that have defined global policies (Barandiarán, 2017), of which cooperation and education constitute two objects of analysis and research. To this end, we review the Spanish case of the ODA-education tandem and how, closely linked to the homogenizing world political orthodoxy that is intentionally expressed by international organizations, ODA at present and in this specific area has been channelled and transmuted into the fourth Sustainable Development Goal (ODS 4), also focused on education. Qualitative techniques are used to analyse documents and content of international organizations and official reports on ODA and SDG 4. The conclusions highlight the initial discourse of the existing link between current Spanish policy on SDG 4 and that of ODA, while elucidating its most immediate future projection.

Keywords: development cooperation; education; official development assistance; Sustainable Development Goals; international education

\author{
Sumario \\ 1. Introducción \\ 4. Las cifras hablan: análisis de la \\ 2. AOD y educación: alianzas \\ situación española con respecto al ODS 4 \\ para el desarrollo en España \\ 3. Cuando los organismos multilaterales \\ 5. Conclusiones: ODS 4, educación y \\ cooperación internacional en España \\ encontraron al ODS 4 Referencias bibliográficas
}

\title{
1. Introducción
}

La ayuda oficial al desarrollo (AOD) constituye «el centro neurálgico de la política de cooperación al desarrollo española» (Martínez-Usarralde, 2011, p. 103). Partiendo de esta premisa, una definición oficial la identifica como una parte de la cooperación internacional para el desarrollo consistente en la ayuda financiera y técnica acordada por el sector público de un país desarrollado, con el objeto de promover los países en vías de desarrollo. De acuerdo con dicha definición, e indagando en su origen y etiología, fue el organismo de Naciones Unidas quien, en 1972, a partir de una recomendación que surgió en una conferencia previa sobre comercio y desarrollo, interpeló a los países para que 
destinaran una cantidad equivalente al $1 \%$ de su PIB (producto interior bruto) a los países en desarrollo, cantidad esta que, a posteriori, se decidiría que quedaría redistribuida en dos partidas: un $0,7 \%$ para las administraciones públicas estatales y un 0,3 para los países receptores.

Unido a lo anterior, además, tal y como apostilla Bindé (2006) a partir de cálculos en un informe emitido por la UNESCO, en la actualidad haría falta un $6,5 \%$ del PIB mundial para acabar con la brecha económica que distancia a los países desarrollados y a los que se encuentran en vías de desarrollo. En esta línea, los diversos informes emitidos a partir de la información obtenida desde la ortodoxia en materia de AOD siguen arrojando cifras poco alentadoras respecto a dicha recomendación: se sigue ratificando que un número muy limitado de países la cumplen o incluso la superan (Luxemburgo, Suecia, Reino Unido, Bélgica y Holanda) y muestran un discreto $0,50 \%$ global como cifra media que atestigua el valor de la AOD en el mundo actual con los datos correspondientes más recientes (European Commission, 2018, p. 1).

Con estas premisas, el presente artículo pretende centrar su atención en la política sectorial española de la AOD vinculada a la educación desde la ingeniería cosmopolita de los objetivos de desarrollo sostenible que han permitido, en palabras de Barandiarán (2017), odeseizar las políticas mundiales, de las que la cooperación y la educación constituyen dos elementos insoslayables y, por ende, objeto de análisis e investigación. Para ello, sus objetivos se centrarán, en primer lugar, en revisar el caso español del binomio formado por AOD y educación. En segundo lugar, en ver cómo, estrechamente vinculado a la ortodoxia política mundial homogeneizadora que proviene de manera intencionalmente manifiesta por parte de los organismos internacionales como el Banco Mundial y la UNESCO, entre los principales (Martínez-Usarralde y Viana-Orta, 2018), la AOD en la actualidad y en este ámbito específico se ha canalizado y ha transmutado en el cuarto objetivo de desarrollo sostenible, centrado también en la educación. De todo lo anterior se deriva, a modo de conclusión, la radiografía a la situación española en torno a la configuración del ODS 4 en educación y cooperación.

\section{AOD y educación: alianzas para el desarrollo en España}

A nivel nacional, España ocupa el décimo lugar entre los países del CAD (Comité de Ayuda al Desarrollo) por el volumen de fondos aportados a la educación en el último decenio (OECD-STATS, s. d.) y es considerado un país pionero por ello, conjuntamente con la prioridad otorgada a sanidad, agua e igualdad de género (Villena, 2017). A pesar de que el compromiso del 0,7\% resultara precisamente ratificado por este país en la declaración final de la Tercera Cumbre sobre la Financiación del Desarrollo y Agenda de Acción de Addis Abeba adoptada en 2015, la Coordinadora Española de Organizaciones no Gubernamentales señala que la AOD presupuestada se estanca, según las cifras más actuales, en el 0,21\% (CONGDE, 2017), situándose muy por debajo de la media del $0,51 \%$ de la UE15 y del 0,32\% de los países de la 
OCDE, lo que le coloca a la cola de los países donantes. De ahí que esta materia se identifica con un reto concreto: alcanzar el compromiso de solidaridad adquirido formalmente y ascender al 0,4\% (CONGDE, 2017). El Ministerio de Asuntos Exteriores y de Cooperación (2018), responsable del V Plan Director de Cooperación Española 2018-2021, por su parte, en este sentido se compromete a "superar una visión más clásica de la AOD» (Ministerio de Asuntos Exteriores y de Cooperación, 2018, p. 11), para lo que se apremia a «revisar sus métodos, criterios y mediciones que recoja en toda su amplitud aquellas innovaciones» (Ministerio de Asuntos Exteriores y de Cooperación, 2018, p. 11). No en vano, la evaluación se ha convertido en una irremisible seña de identidad de las líneas programáticas de la cooperación española, a juzgar por el hecho de que uno de los documentos más descargados en materia de cooperación española en el año 2017 fue el Informe anual de evaluación 2017 (Ministerio de Asuntos Exteriores y de Cooperación, 2017).

La intención explícita de incrementar la AOD en los próximos ejercicios «como muestra del compromiso de España con la cooperación internacional para el desarrollo y el cumplimiento con los ODS» (Ministerio de Asuntos Exteriores y de Cooperación, 2018, p. 62), conjuntamente con la publicación de datos estadísticos de la AOD española a través de Info@OD y el compromiso con el mantenimiento de los programas de ayuda humanitaria con la población saharaui y siria refugiadas marcan las líneas programáticas actualmente trazadas por el Gobierno español.

Siguiendo ahora, por tanto y dentro del caso español, con la atención concedida a la educación, es el V Plan Director de Cooperación Española (2018-2021) el que aporta el marco político que justifica la ayuda en este ámbito y desde el que se señala que:

A pesar de los importantes avances producidos en el acceso a la educación a todos los niveles, el logro de una educación universal, gratuita, inclusiva y equitativa de calidad sigue siendo una tarea pendiente clave para la erradicación de la pobreza y el pleno disfrute de derechos. (Ministerio de Asuntos Exteriores y de Cooperación, 2018, p. 14)

De acuerdo con lo anterior, en España se destina el 5\% de la AOD educativa, como puede contemplarse en la figura 1 .

Se trata ciertamente de un porcentaje alto comparado con «servicios sociales», "reconstrucción de paz» o "género", por ejemplo, y solo superado por "gobierno, sociedad civil y derechos humanos» (un 8\%), aunque queda un apartado, "otros sectores», que detenta la mitad y no ha sido desarrollado.

Ligado a lo anterior, y habiendo hecho además referencia a ello previamente, ¿̇con qué sectores se vincula la AOD como objeto en materia educativa, desde los apartados que se han considerado a efectos de organizar y gestionar la ayuda bilateral? La figura 2 da respuesta de ello.

En esta ocasión, la "educación primaria», a la que se une la "educación de primera infancia», con un $13 \%$, no ocupa el primer puesto como la literatura 
Figura 1. Sectores de cooperación española (2015)

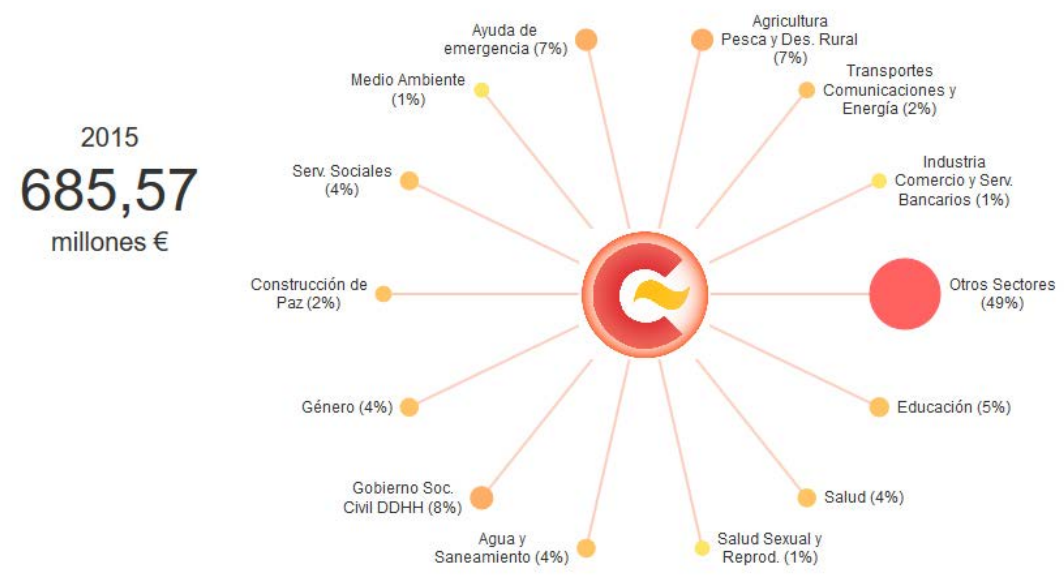

Fuente: Cooperación en Cifras Exteriores, s. d. Recuperado el 11 de abril de 2019, de <http://cooperacion encifras.exteriores.gob.es/es-es/sectores/Paginas/default.aspx>.

Figura 2. Distribución por sectores de la AOD bilateral bruta en educación (2015)

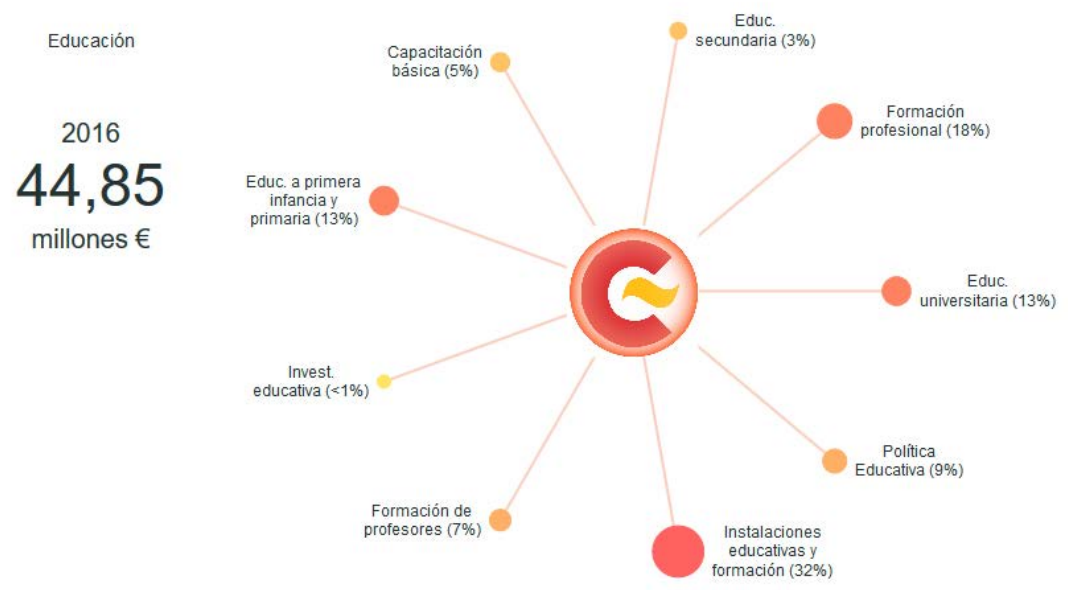

Fuente: Cooperación en Cifras Exteriores, s. d. Recuperado el 11 de abril de 2019, de <http://cooperacion encifras.exteriores.gob.es/es-es/sectores/Paginas/default.aspx>.

especializada señala como tendencia global internacional, sino que les preceden «instalaciones educativas y formación» (32\%) y «formación profesional» (18\%). La «educación universitaria» alcanza, en este caso, el mismo porcenta- 
je que la educación primaria e infantil. Lejanos quedan, en este sentido, tanto la «educación secundaria» (3\%) como la «investigación educativa» $(-1 \%)$.

Cifras como las expuestas en las líneas de arriba, así como todas sus especificaciones, han constituido el objeto de atención y crítica por parte de ONGD (como la que se produce a partir de la coalición entre Entreculturas, Alboan y la Fundación Etea para el Desarrollo y la Cooperación), las coordinadoras de ONGD, presididas por la Coordinadora Española de ONGD (CONGDE), que publica anualmente su informe al respecto, así como análisis surgidos desde la academia universitaria acerca de esta temática a lo largo de los últimos decenios (Martínez-Usarralde, 2011; Mesa, 1995; Ortega, Hernández y Torres, 2006).

En efecto, de sus respectivos análisis cabe considerar que, con carácter general, el sector educativo constituye uno de los principales receptores de fondos de la AOD española bilateral, como lo muestran los análisis realizados sincrónicamente (Martínez-Usarralde, 2011, p. 108), si bien desde una óptica diacrónica, de acuerdo con los informes más recientes consultados, «esta disminuyó por primera vez en 2008 y, aunque aumentó en los dos años siguientes, alcanzando en 2010 su máximo histórico con 13 mil millones de dólares, volvió a caer en 2011, 2012 y 2013» (Entreculturas, Alboan y Fundación Etea para el Desarrollo y la Cooperación, 2017, p. 20), lo que acredita una involución que no ayuda a consolidar la tendencia educativa (Entreculturas, Alboan y Fundación Etea para el Desarrollo y la Cooperación, 2008 y 2017). Esta predisposición a la baja no se corresponde, para concluir en este punto, con la propensión generalizada, ascendente, de la AOD bilateral total.

Con respecto a los sectores que se han priorizado en educación, resulta interesante comprobar la comparativa diacrónica que se centra en valorar, de nuevo, la evolución de cómo se han distribuido sectorialmente los temas educativos en materia de la ayuda (Entreculturas, Alboan y Fundación Etea para el Desarrollo y la Cooperación, 2017, p. 40-41), distinguiendo hasta cuatro etapas:

- Una primera etapa (2000-2006), en la que se prioriza la ayuda destinada a la educación postsecundaria, llegando a duplicar a los destinados a la educación básica (327 millones de euros frente a 183).

- Una segunda etapa (2007-2010), en la que se invierte la tendencia anterior y se destinan a la educación postsecundaria menos de la mitad de recursos que a la educación primaria (195 millones frente a 437).

- Una tercera etapa (2011-2014), caracterizada por la caída global de los recursos y, por consiguiente, por el descenso de ayuda a todos los niveles educativos.

- Finalmente, la última etapa, en la que por primera vez las prioridades son, como se han indicado, otras: instalaciones educativas y formación profesional.

Para concluir, en estos años han recibido ayuda en educación por parte de la cooperación española un total de 138 países, aunque solo en 29 de ellos ha sido por un importe igual o superior al $1 \%$ del apoyo total. Entre las regiones 
receptoras del $80 \%$ de la ayuda española destinada a la educación destacan, por orden (para el periodo 2000-2012): América Latina (53\%), África Subsahariana $(20 \%)$ y, a distancia, África del Norte (9\%) (Ministerio de Asuntos Exteriores y de Cooperación - PACI, 2017). Por países, Marruecos, Brasil, Bolivia, Guatemala, Haití y Perú ocupan los primeros puestos.

\section{Cuando los organismos multilaterales encontraron al ODS 4}

Tras la asistencia exterior recibida después de la Segunda Guerra Mundial desde las intenciones de reconstruir, influir políticamente y también desde valores de altruismo, la ayuda internacional comenzó con el Plan Marshall de los Estados Unidos desde 1948 hasta 1952 para la reconstrucción de 14 países europeos, de los cuales Inglaterra y Noruega fueron los que recibieron mayores porcentajes (Moyo y Pipino, 2009). A partir de ese primer momento, las organizaciones multilaterales fueron forjando una política supranacional de ayuda internacional que combinaba la política y los motivos humanitarios hasta llegar a tener el peso estratégico actual en materia de cooperación al desarrollo internacional. UNESCO, UNICEF, el Banco Mundial y la OCDE, a través de su fondo particular — el CAD—, constituirían los principales agentes, además de otros bancos de desarrollo (influyendo, por este orden, el americano, el británico y el japonés) y la propia Unión Europea ${ }^{1}$.

Desde un punto de vista comparado, ha sido precisamente el Banco Mundial el que, desde una perspectiva diacrónica, le ha asignado una mayor proporción, seguido por la Unión Europea, lo que, en términos porcentuales con respecto a la dimensión total de los presupuestos para cooperación, la ayuda a la educación supone un 4\% para el caso del Banco Mundial y un 5,8\% para la UE de media (Heyneman y Lee, 2016, p. 10).

Del análisis de las alianzas entre la multilateralidad en cooperación, de alcance mundial, en los que destacan con luz propia el Banco Mundial, la UNESCO, la Unión Europea y la OCDE, se desprende, tal como sostiene Mundy (1998), que los organismos pierden parte de su idiosincrasia en este nuevo pacto mundial:

[...] el Banco Mundial habla cada vez más de empoderamiento, mientras que UNESCO, por su parte, lo hace sobre pobreza y subdesarrollo, además de aliarse con sectores privados en materia educativa al ofrecer asistencia y experticia técnica y guía política, con un rol cada vez más rotundo a través de la advocacy (reivindicación de derechos) y de la promoción programática de su ética de valores. (Martínez-Usarralde y Viana-Orta, 2018, p. 26-27)

Además, y por otro lado, el acceso, que podría interpretarse incluso como democratización de los informes y de los estudios que son comparados per se,

1. Sin embargo, las cifras más actuales de que se dispone, correspondientes a 2016 (OECD, 2018a), sin revertir esta situación, sitúan a la Agencia Alemana de Cooperación al Desarrollo incluso por delante del primer organismo. 
como lo demuestran los acometidos desde organismos supranacionales como la Unión Europea, la UNESCO — a través de sus Global Education Monitoring Report (GEM) — o la OCDE — con los estudios PISA—, ha provocado su difusión y divulgación, que, aun siendo positiva, comporta peligros como el de que la comparación se utilice con fines puramente intervencionistas sin analizar de manera pormenorizada contextos que marcan su identidad.

Lo anterior genera la emergencia tanto de detractores como de férreos defensores de estos modelos homogeneizadores que, hoy por hoy, marcan la ortodoxia de la política mundial (King, 2007; Sobe, 2015). De un modo u otro, estos organismos han permitido erigir una suerte de vulgata discursiva, tal y como la definen Nóvoa y Yariv-Mashal (2003), que ratifican la globalidad de la comparación y que, a través de cifras, legitiman la garantía de la calidad y la gestión pública. Lo hacen a través, además, de otros dos elementos: por un lado, contando con políticas de externalización (Popkewitz, 2004) para justificar por qué naciones particulares usan políticas extranjeras o globales para certificar lo que se hace localmente, y, por otro lado, sirviéndose de un concepto, el de gobernanza, cada vez menos nítido y con el que, según Normand (2016), trata de compensar el desencanto creado por el déficit democrático que supone apostar precisamente por la implementación de esta lógica de evaluación comparativa plasmada en las listas de clasificación educativa.

En este mundo globalizado se universalizan también, en efecto, los debates educativos como consecuencia del escenario mundial presentado, tal y como lo prueba quizá su ejemplo hoy y ahora más paradigmático: la hoja de ruta que acredita la denominada Agenda 2030 y los objetivos de desarrollo sostenible (ODS). Reflejan con ello, un nuevo régimen de gobernanza educativa global (Tikly, 2017), sostenido en el proyecto previo de Educación para Todos (EFA), que ocupó desde la década de 1990 hasta enlazar con la citada actual agenda 2030, cuyo centro neurálgico lo constituye la educación para el desarrollo sostenible.

A partir de un marco definido desde cinco esferas de vital importancia (conocidas como las 5 P): personas (people), planeta (planet), paz (peace), prosperidad (prosperity) y alianzas (partnership); la Agenda 2030, "civilizatoria, holística, indivisible y universal» (CEPAL, 2017) articula los 17 ODS «desde el puzzle de la sostenibilidad» (Moya, 2017), de manera que, desde su eje programático:

[...] la Agenda 2030 es un plan de acción en favor de las personas, el planeta y la prosperidad. También tiene por objeto fortalecer la paz universal dentro de un concepto más amplio de la libertad. Estamos resueltos a liberar a la humanidad de la tiranía de la pobreza y las privaciones, y a sanar y proteger nuestro planeta. También se pretende hacer realidad los derechos humanos de todas las personas y alcanzar la igualdad entre los géneros y el empoderamiento de todas las mujeres y niñas. (ONU, 2015a, p. 1)

Desde este escenario, la Agenda Educativa 2030 constituye «una oportunidad para que imaginarios sociales transformacionales sean el punto de par- 
tida para repensar visiones, estrategias y contenidos educativos, así como alinear las piezas de un sistema educativo con dichos imaginarios» (Oficina Internacional de Educación, 2017, p. 7). Se trata, así, de tener de nuevo la posibilidad (una ventana de oportunidades, de hecho, tal y como sostiene la OIE) de repensar la educación como agente de cambio y desde una visión humanística, holística y progresista del desarrollo en sus finalidades, objetivos, estrategias e instrumentos, así como todo el impacto que pueda tener en la organización y el funcionamiento del sistema educativo. Se insiste, de ese modo, en el poder que subyace en la agenda de conceder un renovado sentido y contenido a la construcción colectiva de la educación que ha tenido lugar en los últimos años, reposicionando a esta como indiscutible agente de cambio a través del ODS 4. Y coloca con ello el debate educativo a un nivel alto de exigencia de un diálogo genuinamente plural, así como de una construcción abierta con la ciudadanía, la sociedad civil y el sistema político, haciendo a todos ellos corresponsables de la educación.

La formulación de este objetivo sigue la estela de las aspiraciones marcadas en el Foro Mundial de Educación celebrado en Incheon (Corea) (UNESCO, 2015), si bien, desde 1990 y de manera progresiva, se ha ido conformando una cosmovisión socioeducativa trasmutada en una agenda más específica en materia de educación que, ineludiblemente, remite al Foro Mundial de Educación de Dakar y a la iniciativa de Educación para Todos (EPT). Desde la consideración de cómo ha de seguir constituyendo el marco más amplio de referencia, resulta notorio reconocer que el ODS 4, vinculado a la educación, consigue avances considerables a destacar en relación con lo que se establecía en los ODM. El ODS 4 trae consigo un cierto cambio de enfoque, asentado en dos elementos fundamentales (Entreculturas, Alboan y Fundación Etea para el Desarrollo y la Cooperación, 2017; Moya, 2017): por una parte, su intento de dotarse de un carácter más integral que el que presentaban los ODM, de tal forma que se garantiza la educación básica para todos los colectivos humanos (UNESCO, 2017b). Bajo la aspiración de que los objetivos planteados no se circunscriban al ámbito de la educación primaria, sino que sean capaces de atender a los diversos ciclos y etapas vitales que atraviesan las personas, se incluye desde la educación infantil hasta la educación superior, pasando por la alfabetización de jóvenes y personas adultas y la formación técnica y profesional. Y, por otra, la agenda 2030 parece conceder una mayor importancia a aspectos relativos a la calidad, primándola frente al mero acceso y a las tasas de escolarización que como se demostró prevalecieron en los ODM, acotándose además, de manera casi exclusiva, a la educación primaria. Frente a esta visión, en la Agenda 2030 se otorga más relevancia a cuestiones como el aprendizaje a lo largo de la vida, la equidad, la igualdad de género y la inclusión de los sistemas educativos. 


\section{Las cifras hablan: análisis de la situación española con respecto al ODS 4}

España, como otros países del mundo en este momento, ha de leer (odeseizar) todos los parámetros de política internacional, de la que la ayuda oficial al desarrollo y, en concreto, la destinada a la educación, va a centrar nuestro interés desde el análisis acometido en este estudio. La metodología utilizada se basa en técnicas cualitativas centradas en el análisis documental y de contenido sobre AOD y ODS 4 procedente del Gobierno de España (2018a), Eurostat (2018), OECD (2018b) y SDG Index and Dashboards Report 2018.

En 2014 el Gobierno de España, con el fin de promover el desarrollo sostenible por medio de programas conjuntos de carácter integral y multidimensional, contribuyó inicialmente a crear el Fondo para los Objetivos de Desarrollo Sostenible (Fondo ODS-SDGF) ${ }^{2}$ de la ONU, apoyando con ello programas de desarrollo sostenible a través de programas conjuntos multidimensionales. Actualmente este Fondo ODS gestiona programas conjuntos en 22 países que están mejorando las vidas de más de 5,4 millones de personas ${ }^{3}$ y que se centran en el crecimiento económico inclusivo para la erradicación de la pobreza, la seguridad alimentaria, la nutrición y el agua y el saneamiento. En la mayoría de dichos programas, la educación constituye una base para su fundamentación. En la misma línea, en 2018 creó el Alto Comisionado para la Agenda 2030, con la función de impulsar y desarrollar planes y estrategias, evaluar, verificar y difundir su avance.

En lo que respecta ahora al ODS 4, frente a este postulado que ratifica la tendencia internacional, España se enfrenta, en esta materia, con importantes desafíos a nivel interno, tal y como muestra un número remarcable de informes internacionales que revelan la existencia de un remarcable conjunto de debilidades y deficiencias en el ámbito de la educación: gasto público en educación por debajo del promedio de países de la OCDE, niveles de rendimiento educativo notablemente menores que los que tienen los países de la UE y la OCDE, así como tasa de abandono escolar temprano duplicada, entre otros (Eurostat, 2018; OECD, 2018b).

A esta realidad interna se suma, de facto, la política exterior que quiere extender en materia de AOD, para lo que, a continuación, se presentan los datos comparados de la asistencia para el desarrollo destinada a la educación bilateral y multilateral por parte de España, Francia, Eslovenia, República Checa e Italia, según datos del GEM-Global Education Monitoring (UNESCO, 2019). Se toman como referencia estos países europeos por mostrar un índice de desarrollo humano similar al español, según datos del Programa de las Naciones Unidas para el Desarrollo (2018) (tabla 1).

2. Recuperado el 11 de abril de 2019, de <http://www.sdgfund.org/es>.

3. Recuperado el 11 de abril de 2019, de <http://www.sdgfund.org/es/programas>. 
Tabla 1. Índice de desarrollo humano (IDH)

\begin{tabular}{clc}
\hline Clasificación según el IDH & \multicolumn{1}{c}{ País } & IDH \\
\hline 23 & Francia & 0,901 \\
24 & Eslovenia & 0,896 \\
25 & España & 0,891 \\
27 & República Checa & 0,888 \\
28 & Italia & 0,880 \\
\hline
\end{tabular}

Fuente: Programa de las Naciones Unidas para el Desarrollo (2018).

Francia se mantiene como quinto mayor donante de ayuda a nivel mundial, por detrás de Estados Unidos, Alemania, Reino Unido y Japón. La AOD destinada a la educación por parte del Gobierno francés supera ampliamente a la de España (tabla 1), siendo cinco veces superior en 2005 y veinte veces superior en 2016. Los esfuerzos por parte del Gobierno francés se dirigen mayoritariamente a la educación postsecundaria, mientras que, en cambio, el Gobierno español centra sus intervenciones tanto en educación básica como en secundaria y postsecundaria. Francia presenta, además, un modelo de AOD muy comprometido e innovador con cinco retos, uno de ellos centrado en la educación y que consiste en redoblar esfuerzos para avanzar en todas las etapas educativas, conjuntamente con la investigación y la innovación.

En el caso esloveno y checo no se presentan datos significativos. Finalmente, cabe destacar que Italia (tabla 2), cuya AOD destinada a educación estuvo por debajo de la española en 2005, en 2016 destinó casi el doble que la AOD española y que la mayor proporción fue para la educación básica y postsecundaria y menor para la secundaria.

Tabla 2. Asistencia para el desarrollo destinada a la educación por donante

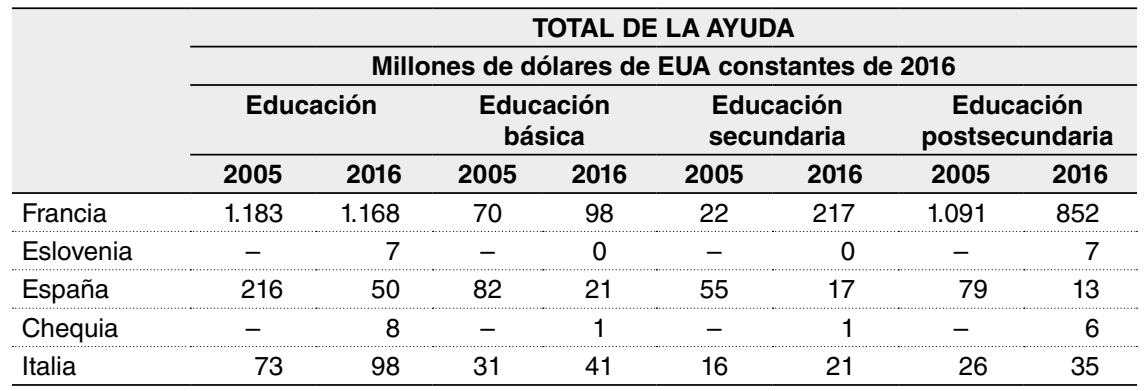

Fuente: CAD de la OCDE, base de datos del OECD CRS (2018).

Unido a lo anterior, el Gobierno de España publicó el Plan de Acción para la Implementación de la Agenda 2030, documento orientado a la ejecución que, en primer lugar, realiza un diagnóstico del estado de cada ODS a nivel nacional y, a continuación, propone una serie de medidas orientadas a la acción. En él, el ODS 4, Educación de Calidad, busca garantizar una educación inclusiva y equitativa de calidad y promover oportunidades de aprendi- 
zaje permanente para todas las personas. En el diagnóstico que realiza el Gobierno de España (2018b) en este plan de acción señala que resulta de vital importancia fomentar una educación para el desarrollo sostenible y la educación para la ciudadanía global, la educación intercultural y la educación para la comprensión internacional. Por este motivo, es necesario potenciar el papel de la educación en el cumplimiento de los derechos humanos, la paz, el ejercicio responsable de la ciudadanía local y global, la igualdad de género, el desarrollo sostenible y la salud.

El SDG Index and Dashboards Report 2018 (Sachs et al., 2018), por su parte, publica datos relativos al cumplimiento de cada país en relación con los ODS. Este informe permite sentar una línea base sobre dónde estamos al señalar temas que merecen atención y en cuyo seguimiento los países deben invertir para seguir avanzando. A continuación nos centraremos en los datos relativos al ODS 4, que serán completados con el Informe de España para el examen nacional voluntario 2018 sobre los ODS (Gobierno de España, 2018a).

Figura 3. Rendimiento general de España en los ODS con respecto a los países de la OECD

\section{SPAIN}

\section{OECD Countries}

\section{$\boldsymbol{\nabla}$ OVERALL PERFORMANCE}

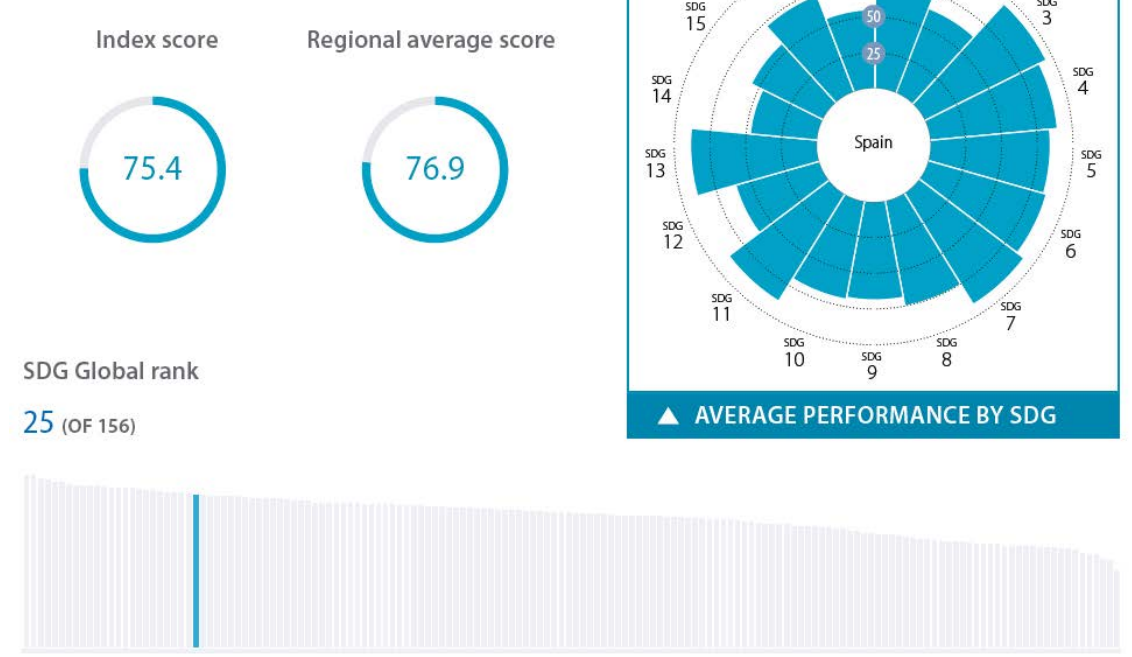

Fuente: SDG Index and Dashboards Report 2018. 
En la figura 3 se presenta el rendimiento general de España en los ODS con respecto a los países de la OECD. En relación con el ODS 4, España alcanza un rendimiento medio que supera el $75 \%$, aunque detenta algunos aspectos a mejorar. La figura 4 muestra datos relativos al ODS 4 y se observa un buen rendimiento en la tasa neta de matriculación en primaria, en la tasa de alfabetización de jóvenes de 15 a 25 años de ambos sexos y en la población de 25 a 64 años con educación terciaria. Entre los aspectos a mejorar dentro del ODS 4 destacan los años promedio de escolaridad: España viene arrastrando una tasa de abandono educativo temprano ligeramente superior con respecto al resto de países de la OCDE, aunque, tal y como indica el Gobierno de España (2018a), se ha reducido en los últimos años, pasando del 26,3\% en 2011 hasta el 18,3\% en 2017.

Figura 4. Análisis del ODS 4 en España

SDG4 - Quality Education

Net primary enrolment rate (\%)

Mean years of schooling

Literacy rate of $15-24$ year olds, both sexes (\%)

Population age 25-64 with tertiary education (\%)

PISA score (0-600)

Variation in science performance explained by students' socio-economic status (\%)

Students performing below level 2 in science (\%)

Resilient students (\%)

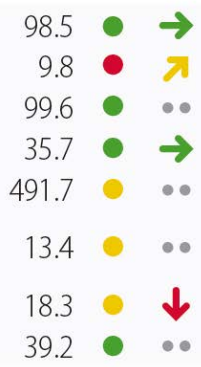

Fuente: SDG Index and Dashboards Report 2018.

\section{Conclusiones: ODS 4, educación y cooperación internacional en España}

¿Qué respuesta, en materia de política nacional e internacional, puede emprender la Administración con la perspectiva de alcanzar esta meta? Partiendo de las recomendaciones y de las acciones recogidas en la Declaración de Incheon y del Marco de Acción para la Educación 2030 de la UNESCO, de la investigación La ayuda en educación a examen, fruto de la coalición formada por las organizaciones Entreculturas, Alboan y Fundación Etea para el Desarrollo y la Cooperación (2017), y de los informes emitidos por el Gobierno de España (Gobierno de España, 2018a y 2018b), las estrategias propuestas para alcanzar la meta 4.1 que podrían aplicarse en nuestro país deberían traducirse en un triple frente: definir normas y analizar planes de estudio para garantizar su adecuación al ODS 4; asignar recursos de manera más equitativa, a fin de conseguir una mayor inclusión educativa y crear sistemas de evaluación formativa, con el objetivo de retroalimentar el proceso que reflejen las capacidades cognitivas y no cognitivas adquiridas por el alumnado. 
En relación, finalmente, con las políticas internacionales, la UNESCO (2015) interpela a los países desarrollados que aún no alcanzan la meta de destinar el $0,7 \%$ del PNB a AOD o a dedicar más esfuerzos para conseguirlo. Además, recomienda mejorar la eficacia de la ayuda mediante una mejor coordinación y armonización para conseguir garantizar el derecho a la educación para todas las personas, con mayor insistencia en situaciones de crisis humanitarias. Por último, incide en la elaboración de informes de seguimiento sobre el ODS 4. Nos hallamos, por todo ello, en proceso de conseguir en España un ODS 4 integral, inclusivo y equitativo, sostenible y vinculado al desarrollo humano y de las capacidades.

\section{Referencias bibliográficas}

BARANDIARÁn, M. (2017). La Universidad como agente en la promoción del desarrollo humano. E-dhc, 8, 44-55.

BINDÉ, J. (2006). Hacia las sociedades del conocimiento: Informe Anual de la UNESCO. París: UNESCO.

CEPAL (2017). Informe anual sobre el progreso y los desafios regionales de la Agenda 2030 para el Desarrollo Sostenible en América Latina y el Caribe. Santiago: CEPAL.

CONGDE (2017). La Ayuda Oficial al Desarrollo en los presupuestos generales del Estado. Madrid: Coordinadora de ONGD para el Desarrollo de España.

Entreculturas, Alboan y Fundación Etea para el Desarrollo y la CooperaCión (2008). AOD en educación a examen: Análisis de la Cooperación Española 2005-2006. Madrid: Entreculturas, Alboan y Fundación Etea.

- (2017). AOD en educación a examen: Balances y retos de la cooperación española en el marco de la agenda 2030. Madrid: Entreculturas, Alboan, Fundación Etea.

European Commission (2018). Publication of preliminary figures on 2017 Official Development Assistance: MEMO/18/3081. Luxemburgo: European Commission.

Eurostat (2018). Education Administrative Data from 2013 onwards. ISCED 2011: Euro SDMX Metadata Structure (ESMS). Luxemburgo: European Commission.

Gobierno de españa (2018a). Objetivos de Desarrollo Sostenible. Informe de España para el examen nacional voluntario 2018. Madrid: Gobierno de España.

- (2018b). Plan de acción para la implementación de la Agenda 2030. Madrid: Gobierno de España.

Heyneman, S.P. y Lee, B. (2016). International organizations and the future of education assistance. International Journal of Educational Development, 48, 9-22. $<$ https://doi.org/10.1016/j.ijedudev.2015.11.009>

King, K. (2007). Multilateral agencies in the construction of the global agenda on education. Comparative Education, 43, 377-391.

Martínez-UsarRalde, M.J. (2011). ¿Cooperación o ayuda?: Análisis comparado de la política de cooperación internacional al desarrollo española en educación en América latina. Contextos Educativos: Revista de Educación, 14, 101-118. $<$ http://dx.doi.org/10.18172/con.642>

Martínez-Usarralde, M.J. y Viana-Orta, M.I. (2018). The Life Cycle of UNESCO Education Policies: Fields, Programmes and Strategies. En M. AKI (ed.). Unesco: Current Issues and Challenges (pp. 25-48). Nueva York: Nova Science Publishers. 
Mesa, M. (1995). Otras formas de cooperar: Presión política y educación. Papeles, 55. Zaragoza: Centro de Investigaciones para la Paz (CIP). Fundación Hogar del Empleado.

Ministerio de Asuntos Exteriores y de Cooperación (2017). Informe anual de evaluación 2017. Madrid: Ministerio de Asuntos Exteriores y de Cooperación.

- (2018). V Plan Director de Cooperación Española 2018-2021. Madrid: Ministerio de Asuntos Exteriores y de Cooperación.

Moya, M.A. (2017). La educación: La clave para el desarrollo sostenible. En R. Goycoolea y M. Megías (eds.). Objetivos del Desarrollo Sostenible: Una mirada crítica desde la universidad y la Cooperación al Desarrollo (pp. 67-78). Alcalá: Universidad de Alcalá-COOPUAH.

Moyo, D. y Pipino, E. (2009). Cuando la ayuda es el problema: Hay otro camino para África. Madrid: Gota a Gota.

Mundy, K. (1998). Educational Multilateralism and World (Dis)Order. Comparative Education Review, 42, 448-478.

Normand, R. (2016). The Changing Epistemic Governance of European Education: The Fabrication of the Homo Academicus Europeus? Suiza: Springer.

Nóvoa, A. y Yariv-Mashal, T. (2003). Comparative research in education: A mode of governance or historical journey? Comparative Education, 39(1), 423-438. <https://doi.org/10.1080/0305006032000162002>

OECD (2018a). International Development Statistics (IDS) online databases: Aid (ODA) by sector and donor [DAC5]. Organisation for Economic Co-operation and Development. Recuperado en diciembre de 2018, de <http://stats.oecd.org/Index. aspx?datasetcode=TABLE5>.

- (2018b). Education at a glance. París: OECD.

OECD CRS (2018). Creditor reporting system. Organisation for Economic Co-operation and Development. Recuperado en abril de 2019, recuperado en abril de 2019, de <https://stats.oecd.org/Index.aspx?DataSetCode=CRS1>

OECD STATS (S.D.). Total flows by donor (ODA+OOF+Private) [DAC1]. Organisation for Economic Co-operation and Development. Recuperado en abril de 2019, de <https://stats.oecd.org/>.

Oficina InTERnaCiOnAL de EduCaCión (2017). 15 claves de análisis para apuntalar la Agenda Educativa 2030. Ginebra: OIE.

ONU (2015a). Transformar nuestro mundo: La Agenda 2030 para el Desarrollo Sostenible. A/RES/70/1 (25 de septiembre de 2015 - 21 de octubre de 2015).

- (2015b). Objetivos de Desarrollo del Milenio: Informe de 2015. Nueva York: Naciones Unidas.

Ortega, M.L.; Hernández, A. y Torres, M. (2006). La Ayuda Oficial al Desarrollo bilateral española en educación en el periodo 1999-2004: Perfil y recomendaciones. Revista de Fomento Social, 61, 185-212.

Popkewitz, T. (2004). Foreword. En G. Steiner-Khamsi (ed.). The Global Politics of Educational Borrowing and Lending (pp. 1-9). Nueva York: Teachers CollegeColumbia University.

Programa de las Naciones Unidas para el Desarrollo (2018). Indices e indicadores de desarrollo humano: Actualización estadistica de 2018. Nueva York: PNUD.

Sachs, J.; Schmidt-Traub, G.; Kroll, C.; Lafortune, G. y Fuller, G. (2018). SDG Index and Dashboards Report 2018. Nueva York: Bertelsmann Stiftung and Sustainable Development Solutions Network (SDSN). 
Secretaría General de Cooperación Internacional para el Desarrollo (2016). Estrategia de Educación para el Desarrollo de la Cooperación Española 20072014: Informe completo. Madrid: Ministerio de Asuntos Exteriores y de Cooperación, Secretaría de Estado de Cooperación Internacional y para Iberoamérica y Secretaría General de Cooperación Internacional para el Desarrollo.

- (2017). Seguimiento AOD 2015. Madrid: Secretaría General de Cooperación Internacional para el Desarrollo. Ministerio de Asuntos Exteriores y Cooperación.

Sobe, N.W. (2015). All that is global is not world culture: Accountability systems and educational apparatuses. Globalisation, Societies and Education, 13(1), 135-148. <https://doi.org/10.1080/14767724.2014.967501>

Tiksy, L.P. (2017). The future of Education for All as a Global Regime of Educational Governance. Comparative Education Review, 61, 22-57. <https://doi.org/10.1086/689700>

UNESCO (2015). Educación 2030: Hacia una educación inclusiva y equitativa de calidad y un aprendizaje a lo largo de la vida para todos. Incheon: World Education Forum.

- (2017a). La educación transforma vidas. París: UNESCO.

- (2017b). Rendir cuentas en el ámbito de la educación: Cumplir nuestros compromisos, resumen del informe de seguimiento de la educación en el mundo, 2017/8. París: UNESCO.

- (2019). Migration, displacement and education. Building bridges, not walls. Global Education Monitoring Report-GEM Paris: UNESCO.

Villena, M.A. (2017). España solidaria: 30 años de cooperación española al desarrollo. Barcelona: Ediciones Gestión 2000. 